\title{
Preliminary Study on China's Low-Altitude Airspace Operation Safety Evaluation Method
}

\author{
Qian-Rui Sun ${ }^{1,2, a}$, Deng-Kai Yao ${ }^{1,2, b}$, Yi-Ye Zhou ${ }^{1,2, a}$ \\ ${ }^{1}$ Air Traffic Control and Navigation College, Air Force Engineering University, Xi'an, Shaanxi, \\ 710051, China \\ ${ }^{2}$ National Key Laboratory of Air Traffic Collision Prevention, Xi'an, Shaanxi, 710051, China \\ a811176522@qq.com ${ }^{\mathrm{b}} 737000636 @ q q . c o m^{\mathrm{c}} 13998380516 @ 163 . c o m$
}

\begin{abstract}
Keywords: Low-altitude airspace; Safety factors; Safety evaluation; The Fuzzy Comprehensive Evaluation Method
\end{abstract}

\begin{abstract}
As the innovation of low-altitude airspace is advancing, the operating system is more mature, and the amount of aircraft increases rapidly, resulting in the increasing importance of the operation security. However, today's research on safety evaluation of low-altitude airspace in China is lagging behind. There are some similarities in operation between low altitude airspace and medium/high altitude airspace. With the reference of conventional research of safety evaluation, and combined with the nature and operating characteristics of the low-altitude airspace, safety factors are analyzed and safety evaluation index system is built. Finally, idea and method of safety evaluation is proposed to provide theory basis for identifying the weakness of operation safety in low-altitude airspace.
\end{abstract}

\section{Introduction}

In China, the vertical range of low-altitude airspace is below true height of 1000 meters in principle. Different from medium and high altitude airspace, flight activities in low-altitude airspace usually include general aviation aircraft ${ }^{11]}$, military aircraft, police aircraft and other state aircraft flying at low altitude.With the advancement of China's low-altitude airspace reform, the innovation procedure is entering into a deepening stage. Higher requirements for safety are raised as the scale of low-altitude airspace operation increasing.

Human, machine, environment and management are the core elements in system. All accidents result from defects of human, machine, environment, and management which adjust former three aspects $^{[2]}$. Therefore, the research is launched from these four aspects, safety evaluation index system is constructed accordingly. And the Fuzzy Comprehensive Evaluation Method is presented to evaluate the operation safety.

\section{Safety Factors}

Human is the most active and intractable part in a system. The "see-avoid" principle is usually used for conflict avoidance by aircraft flying at low altitude, so the pilots take major responsibility for safety. Controllers keep the safe interval by supervising the aircraft flying as planned and deploying flight conflict. Operational controllers identify, handle and remove faults timely to ensure facilities run normally. Flight service personnel provide varied information services and alerting service.

Low-altitude aircraft are inevitably affected by environment. China's low-altitude airspace is under dynamic management, thus the use of which is a vital part in safety management. Traffic flow conditions reflect the characteristics of spatio-temporal distribution of the aircraft. Low-level wind shear and other bad weather conditions threaten low-altitude flight safety seriously. Many low-altitude airspace aircraft accidents occurred in China in recent years are caused by obstacles such as unknown ballons,birds and cables, showing that low headroom condition is of great importance to the operation safety.

Management is the link of all elements to work as a whole. Organization and routine face varied operational affairs directly, and is the foundation and strategic part of safety. Laws and regulations 
are the basic principle and important guarantee for operation. Personnel selection and training determines ability of staff, and then operation safety. Information is the record of various states and process, which can help identify the defects of operation and then benefit the safety supervision. Dangerous events can be references to improve operation and management, and prevent the recurrences of similar events. Prevention is to monitor potential risks, evaluate the safety factors periodically, and adjust the undesirable parts to be normal. It aims at eliminating safety hazards, which is the most economical and effective mean to ensure safety.

The machine in low-altitude airspace mainly refers to aircraft and technical equipment in operation. Communication takes the responsibility for all data link between air and ground. Navigation provides aircraft with the flight guidance, according to which a pilot can know his position and fly as planned. Surveillance provides real-time dynamic information of the target and it is the basic of anti-collision and ground targets' protection. Meteorological support provides real-time meteorological information, meteorological forecast and alert information which can help avoid weather triggered hazards. Service acceptance and airworthiness of aircraft influence flight safety directly. Low-altitude flight service station provides meteorological, flight planning, aeronautical information services and alerting service, emergency rescue and other related support ${ }^{[3]}$, as an important guarantee for low-altitude flight safety.

\section{Safety Evaluation}

According to the factors above, build the safety evaluation system as shown in Figure 1.

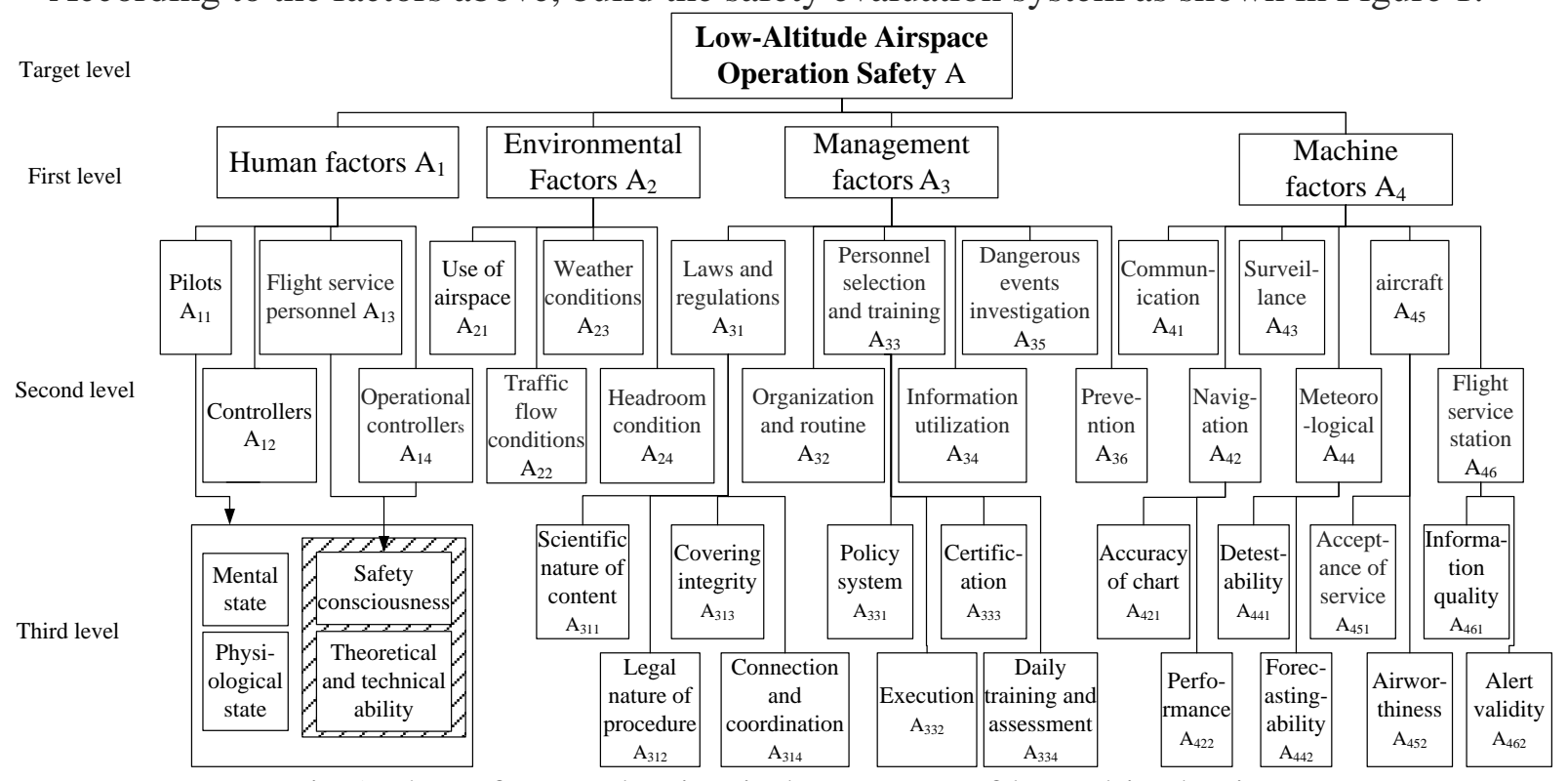

Fig. 1 The safety evaluation index system of low-altitude airspace

Third level indexes related to human factors are: $A_{11}=\left(A_{111}, A_{112}, A_{113}, A_{114}\right)=($ safety consciousness, theoretical and technical ability,mental state, physiological state)(of pilots), $A_{12}=\left(A_{121}, A_{122}, A_{123}, A_{124}\right)$, these four factors related to controllers are same as pilots', $\mathrm{A}_{13}=\left(\mathrm{A}_{131}, \mathrm{~A}_{132}\right)=($ safety consciousness, theoretical and technical ability)(of flight service personnel), $\mathrm{A}_{14}=\left(\mathrm{A}_{141}, \mathrm{~A}_{142}\right)$, these two factors related to operational controllers are same as flight service personnel's.

Based on the Fuzzy Comprehensive Evaluation Method, this paper establishes the qualitative and quantitative analysis of the index system, and provides idea and method of low-altitude airspace operation safety evaluation.

The FCEM is based on fuzzy set theory. Specifically, it helps evaluate the targets' membership grade integrating many factors under fuzzy synthesis principle. The most obvious characteristic of this method is solving qualitative issues with quantitative method which helps overcome the deviation from certainty evaluation with "yes" or "No"[4]. 
Here, the index weight is determined, reviews set is constructed, fuzzy transformation is formed to map the index to reviews set, the operator is fixed and finally the comprehensive evaluation mechanism is presented ${ }^{[5]}$.

Step 1: Determine of the index weight. The AHP is adopted to judge the relative importance of various factors at each level by pairwise comparison. Judgment matrixes are constructed by integer between $1 \sim 9$ and their inverses as scale.

By consulting experts, index judgment matrixes are established. All the judgment matrixes have been shown to pass the consistency check by Matlab programming calculation, and index weight vectors can be obtained: $\quad W A=(0.53,0.34,0.08,0.05)$, $\mathrm{WA}_{1}=(0.10,0.31,0.05,0.54), \mathrm{WA}_{2}=(0.10,0.56,0.23,0.11), \mathrm{WA}_{3}=(0.16,0.39,0.24,0.09,0.07,0.06), \mathrm{WA}_{4}$ $=(0.07,0.08,0.23,0.04,0.45,0.13) ; \mathrm{WA}_{11}=(0.11,0.40,0.28,0.21), \mathrm{WA}_{12}=(0.10,0.42,0.23,0.25), \mathrm{WA}_{13}=(0$ $.20,0.80), \mathrm{WA}_{14}=(0.20,0.80), \mathrm{WA}_{31}=(0.43,0.31,0.21,0.05), \mathrm{WA}_{33}=(0.18,0.27,0.41,0.14), \mathrm{WA}_{42}=(0.66$, $0.34), \mathrm{WA}_{44}=(0.66,0.34), \mathrm{WA}_{45}=(0.5,0.50), \mathrm{WA}_{46}=(0.83,0.17)$.

Step 2: Determine of the reviews set and operator. The reviews set is: V= (excellent, good, medium, low, poor).

Because the generalized operator $(\bullet \oplus)$ has the advantages of obvious effect weight, high integrated degree, sufficient use of R (fuzzy relationship matrix) information, this paper takes it as the operator "०".

$$
b_{j}=\min \left(1, \sum_{i=1}^{m} a_{i} r_{i j}\right)
$$

$a_{i}$ represents the weight of index $i, r_{i j}$ represents the element in row $\mathrm{i}$, column $\mathrm{j}$ of the membership evaluation matrix.

Step 3: Evaluate. In the low-altitude airspace daily operation, safety evaluation is a part of the "prevention" efforts. Reviews of the end indexes are obtained by consulting managers, experts, technical personnel, and based on which the membership evaluation matrixes are built: the element in row $i$, column $j$ represents the percentage of review $j$ in all evaluation results of the element $i$ (the sum of each row element in a matrix is 1 ).

Since some end indexes belong to the second level, such as information utilization and some belong to the third level, the membership evaluation matrix $\left(R_{m n}\right)$ of third level indexes(belong to index $A_{m n}$ ) are built firstly:

$$
R_{m n}=\left[\begin{array}{ccccc}
a_{11} & a_{12} & a_{13} & a_{14} & a_{15} \\
a_{21} & a_{22} & a_{23} & a_{24} & a_{25} \\
\ldots & \ldots & \ldots & \ldots & \ldots \\
a_{s 1} & a_{s 2} & a_{s 3} & a_{s 4} & a_{s 5}
\end{array}\right]
$$

$s$ is the index number of $A_{m n} ; a_{\mathrm{ij}}$ is the percentage of review $j(j=1,2, \ldots .5)$ in all evaluation results of the element $i$. The evaluation vector of $A_{m n}$ is:

$$
B_{m n}=W A_{m n} \circ R_{m n}=\left(b_{m n 1}, b_{m n 2}, b_{m n 3}, b_{m n 4}, b_{m n 5}\right)
$$

The sum of all elements in $B_{m n}$ is not necessarily 1 under the effect of the operator, so normalization process is used to unify evaluation vector:

$$
B_{m n}{ }^{\prime}=\frac{1}{\sum_{i=1}^{5} b_{m n i}}\left(b_{m n 1}, b_{m n 2}, b_{m n 3}, b_{m n 4}, b_{m n 5}\right)=\left(b_{m n 1}{ }^{\prime}, b_{m n 2}{ }^{\prime}, b_{m n 3}{ }^{\prime}, b_{m n 4}{ }^{\prime}, b_{m n 5}{ }^{\prime}\right)
$$

Piece the above evaluation vectors with the membership evaluation vectors made by expert, which belong to indexes without lower indexes at second level, and build membership evaluation matrixes.

$$
R_{1}=\left[\begin{array}{l}
B_{11}^{\prime} \cdot \\
B_{12}^{\prime} \\
\left.B_{13}^{\prime}{ }^{\prime} \cdot\right] \\
B_{14}^{\prime}
\end{array}\right] R_{2}=\left[\begin{array}{l}
B_{21} \\
B_{22} \\
B_{23} \\
B_{24}
\end{array}\right] R_{3}=\left[\begin{array}{l}
B_{31}^{\prime} \\
B_{32} \\
B_{33}^{\prime} \\
B_{34} \\
B_{35} \\
B_{36}
\end{array}\right] R_{4}=\left[\begin{array}{c}
B_{41} \\
B_{42}^{\prime} \\
B_{43} \\
B_{44}^{\prime} \\
B_{45}^{\prime} \\
B_{46}^{\prime}
\end{array}\right]
$$


$A_{i}$ is:

$B_{i j}$ is the evaluation vector of $A_{i j}$ which is the end index at second level. The evaluation vector of

$$
B_{i}=W A_{i} \circ R_{i}=\left(b_{i 1}, b_{i 2}, b_{I 3}, b_{i 4}, b_{i 5}\right)
$$

Membership evaluation matrix of first level is established.

The global evaluation vector is obtained:

$$
R=\left[\begin{array}{l}
B_{1} \\
B_{2} \\
B_{3} \\
B_{4}
\end{array}\right]=\left[\begin{array}{lllll}
b_{11} & b_{12} & b_{13} & b_{14} & b_{15} \\
b_{21} & b_{22} & b_{23} & b_{24} & b_{25} \\
b_{31} & b_{32} & b_{33} & b_{34} & b_{35} \\
b_{41} & b_{42} & b_{43} & b_{44} & b_{45}
\end{array}\right]
$$

$$
B=W A \circ R_{i}=\left(b_{1}, b_{2}, b_{3}, b_{4}, b_{5}\right)
$$

When determining the grade, the principle of maximum degree of membership is generally used. But there is a problem that it is inappropriate to use this principle when some elements in $B$ appear to be same or similar. To avoid this biased evaluation, other methods of determining can be chosen.

Assignment method is used: Recording evaluation results in centesimal system: 0-100 is divided into five intervals respectively corresponding to five grades, and the score of a grade is assigned to its interval value. Interval value and assignment are shown in table1.

Table1 Interval value and assignment

\begin{tabular}{c|c|c|c|c|c}
\hline Grade & Excellent & Good & Medium & Low & Poor \\
\hline Interval & $\mathbf{( 9 0 , 1 0 0 ]}$ & $\mathbf{( 8 0 , 9 0 ]}$ & $\mathbf{( 7 0 , 8 0 ]}$ & $(60,70]$ & $(0,60]$ \\
\hline Assignment & 95 & 85 & 75 & 65 & 30 \\
\hline
\end{tabular}

According to the formula (6), safety status of all index levels and the whole system can be calculated.

$$
Y=B \bullet(95,85,75,65,30)^{\mathrm{T}}
$$

$B$ can be evaluation vector of all levels of indexes or system, and $Y$ represents the corresponding score which can directly reflect the safety status in numerical form, or be mapped to corresponding interval, and evaluation grade according to the above rules can be obtained.

The low-altitude airspace operation safety evaluation should be done at regular time to examine the real-time status of system, so the values in membership evaluation matrixes are got in real-time. A template for evaluating is provided here without specific values in membership evaluation matrixes.

Safety standards of indexes and the overall system should be set according to practical operation. When score or grade is lower than the standard, managers should pay attention and improve the corresponding index to keep the system at a safe status.

\section{Summary}

With the reference of usual altitude airspace, this paper explores the evaluation method of low-altitude airspace combined with its own characteristic which could be theoretical support for its operation safety.

1) Low-altitude airspace operation safety factors are analyzed.

2) The evaluation index system is established, and the evaluation idea and method of low-altitude airspace is presented according to the FCEM.

3) The method of building membership evaluation matrix when end indexes belong to different levels is proposed in fuzzy comprehensive evaluation and the assignment method is designed to calculate the evaluation result or judge the evaluation grade.

\section{Acknowledgments}

This work was supported partly by Provincial Natural Science Foundation Provincial Natural Science Foundation (Grant No. 2015JM7364). 


\section{References}

[1] Y. Yang, D. Sui. Thoughts on low altitude airspace reforming and general aviation development in China[J]. Journal of Nanjing University of Aeronautics and Astronautics, 2012, 12(2), 50-57.

[2] Y. Liang. Air traffic control safety system assessment method based on Bayesian networks[D]. Shenyang: Shenyang Aerospace University, 2010.

[3] S.Z Jin. General aviation flight service station system design and low-level integrated monitoring simulation [D]. Guanghan: School of Traffic Management, Civil Aviation Flight University of China, 2012.

[4] J. Wan, N Li, Q Li. Research on the safety evaluation of air traffic control management system[J]. Journal of Beijing University of Aeronautics and Astronautics (Social Sciences Edition), 2011, 24(1):89-92.

[5] X.Y Xu. Research and application of Fuzzy Comprehensive Evaluation method[D]. Chengdu: Southwest University of Petroleum, 2011. 